\title{
ETAT DES TRAITEMENTS CHIRURGICAUX DE L'IMPUISSANCE
}

\author{
J.P. SARRAMON
}

\author{
Service d'Urologie et Transplantation \\ CHU Purpan - 31059 Toulouse Cedex
}

\section{CURRENT STATUS OF SURGICAL TREATMENT FOR IMPOTENCE. The current status of the various surgical alterna- tives in the treatment of impotence is revie- wed. Microsurgical techniques for arterial l'Impuissance, créé en 1990, par Furlow et Wagner. \\ CHIRURGIE RECONSTRUCTRICE Chirurgie artérielle} revascularization succeed in 70 to $87 \%$ of cases with a mean follow-up of 48 months. In cases of venous incompetence, arterialization of the deep dorsal vein gives the best, most long-lasting results. Penile prostheses have benefitted from continual technological advances with inflatable prostheses providing the best cosmetic appearance. Mechanical failures are now rare, but the cost of prostheses remain high. Vascular surgery continues to be the first option by virtue of its being physiological, and not compromising other procedures such as self-administered injections, vacuum or penile prostheses. Key-words : Vascular impotence, penile prostheses, surgery. Andrologie 1991, 1 : 145-148.

L'impuissance virile a été séparée il y a une quinzaine d'années en impuissance psychogène et impuissance organique. Les méthodes d'exploration récentes et en particulier l'étude des érections nocturnes (NPT) permettent de les différencier. Autrefois, tout dysfonctionnement érectile était attribué à une origine psychogène ou en relation avec le vieillissement (andropause).

Pendant de nombreuses années, le seul traitement chirurgical a été représenté par les prothèses d'abord semi-rigides, qui sont devenues gonflables après de multiples progrès technologiques. Michal (16) en 1973 a proposé des procédés de revascularisation artérielle dans le but d'améliorer les débits artériels intrapéniens. Depuis lors, de nombreuses équipes, surtout européennes, se sont appliquées à découvrir de nouvelles techniques et ce après le ler Congrès International sur l'Impuissance qui se tint à NewYork en 1978. Plus récemment (12), on s'est intéressé à une nouvelle pathologie, celle des incompétences veineuses, sans qu'on puisse pour autant en comprendre exactement le mécanisme.

Tous ces efforts ont permis de mieux cerner les étiologies et de mieux poser les indications thérapeutiques et ceci grâce à des Congrès Internationaux tous les 2 ans, et à un organe com- mun, le Journal International de Recherche sur

Elle est indiquée dans les cas de lésions athéromateuses, de lésions dysplasiques ou post-traumatiques. Celles-ci intéressent parfois les grands axes, mais surtout les branches de division de l'artère honteuse interne. L'indication opératoire est posée à partir d'un examen Doppler sensibilisé par injection de drogues vaso-actives intracaverneuses, mais surtout d'une artériographie hypersélective des artières honteuses internes, pour des patients très motivés, en dessous de 65 ans.

\section{Pontage direct sur les corps caverneux}

Ce fut le procédé initialement décrit et réalisé dans un grand nombre de cas par Michal (16). Il s'agissait d'implanter l'artère épigastrique sur un orifice préalablement ménagé au niveau de l'albuginée, véritable procédé de Vineberg. Cette technique fut progressivement abandonnée pour les risques de priapisme et d'obstruction par fibrose de l'anastomose qu'elle comportait.

\section{Revascularisation de l'artère dorsale}

L'artère épigastrique est pontée en terminolatéral sur l'artère dorsale. Lorsqu'une des deux est perméable, on peut aussi réaliser une anastomose terminoterminale. En général, un seul pontage est nécessaire ; certains en ont proposé deux. Les diamètres artériels sont du même ordre : ils ne dépassent pas $1 \mathrm{~mm}$ à $1,5 \mathrm{~mm}$. C'est le plus classique et le plus utilisé des modes de revascularisation artériels (fig. 1 et 2).

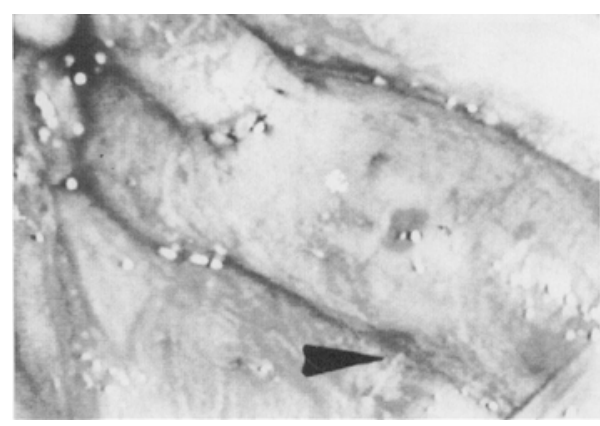

Fig. I : Revalscularisation artérielle à partir de l'artère épigastrique.
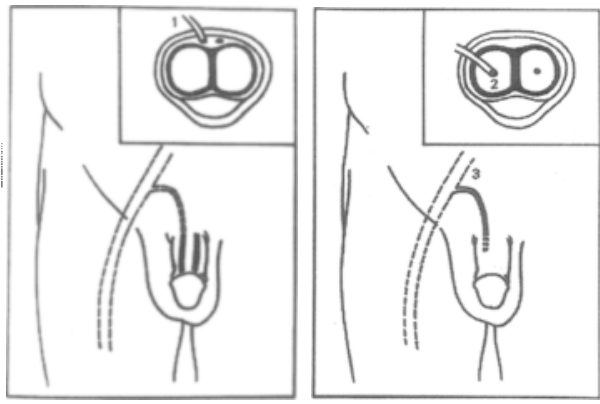

Fig. 2 : Pontage artério-artéric]

\section{Revascularisation des artères profondes}

Il est rare que les artères caverneuses soient potentiellement utilisables. Leur calibre est inférieur à celui des artères dorsales. Toutefois, certains auteurs ont pu revasculariser les artères caverneuses à partir de l'artère épigastrique, ou en réalisant des pontages par des veines antébrachiales entre l'artère fémorale et les artères caverneuses.

\section{Artérialisations de la veine dorsale profonde $(22,23)$}

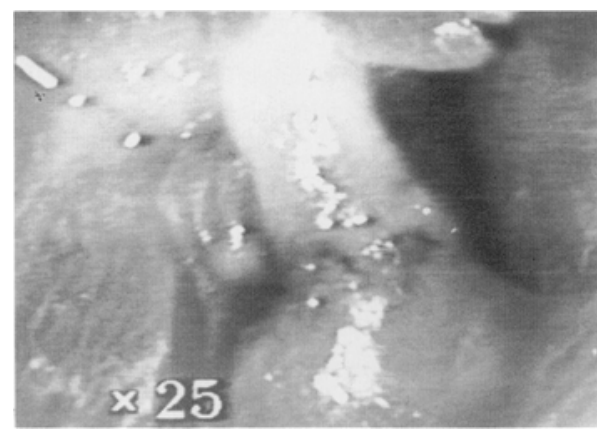

Fig. 3 : Artérialisation de la veine dorsale profonde

Le principe consiste à augmenter le débit artériel dans les sinus caverneux et ralentir le retour veineux ; on n'en comprend pas exactement le fonctionnement. Il convient d'anastomoser l'artère épigastrique sur la veine dorsale profonde en réalisant une fistule artérioveineuse terminolatérale. On dissèque la veine dorsale profonde sur 3 à 5 $\mathrm{cm}$ en recherchant comme site d'anastomose le lieu où elle reçoit 2 à 3 veines émissaires. L'anastomose est le plus fréquemment terminolatérale (fig. 3 et 4 ). On lie la veine dorsale en amont et en aval du pontage pour accroître le débit intracaverneux et prévenir une hypervascularisation du gland. 


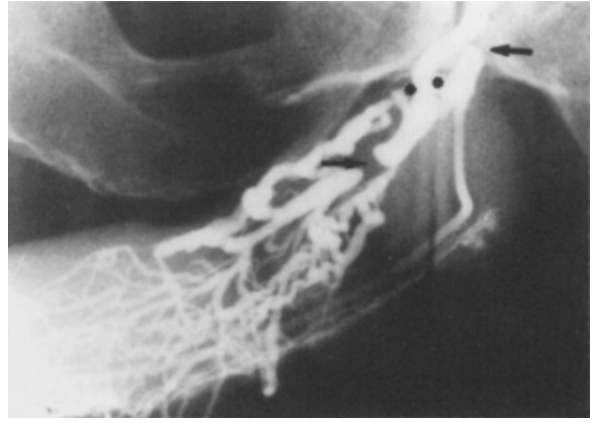

Fig. 4 : Contrôle post-opératoire de l'artérialisation de la veine dorsale profonde

\section{Procédé de Hauri (7)}

Il consiste à réaliser une anastomose entre la veine dorsale profonde et une artère dorsale et à ponter sur cette fistule artérioveineuse l'artère épigastrique.

\section{Chirurgie des incompétences veineuses}

La pathogénie de ce syndrome demeure encore mystérieuse. Les fuites sont souvent associées à des lésions artérielles. Ceci explique certains échecs de la chirurgie artérielle isolée. Pour corriger ces fuites, de nombreuses techniques ont fait la preuve de résultats incomplets et peu durables.

La ligature des veines péniennes a été le premier procédé proposé au début du siècle pour traiter l'impuissance (24), repris avec de multiples variantes. Nous ne les détaillerons pas et nous contenterons d'énumérer les principales :

- ligature de la veine dorsale profonde (24).

- ligature et résection de la veine dorsale profonde et des collatérales émissaires et circonflexes avec le plus souvent une spongiolyse (12).

- ligature complémentaire des veines caverneuses après section des ligaments suspenseurs de la verge (12)

- plicature de la portion fixe des corps caverneux ou intervention Lowsley (13)

- garrot prothétique (17).

\section{Indications}

Elles sont actuellement relativement bien codifiées. Lorsqu'il existe des lésions artérielles pures distales avec une artère dorsale perméable, le meilleur procédé consiste à réaliser un pontage entre l'artère épigastrique et l'artère dorsale. Lorsque les deux artères dorsales sont thrombosées ou de mauvaise qualité, on choisira une artérialisation de la veine dorsale profonde. S'il existe une pathologie mixte, déficit artériel et fuite veineuse associés, chaque fois qu'on le peut, il faut revasculariser les artères dorsales et lier simultanément la veine dorsale profonde et les branches collatérales. Sinon, réaliser une artérialisation de la veine dorsale profonde.
En cas de pathologie veineuse, actuellement avec un recul suffisant, après avoir utilisé comme beaucoup les ligatures multiples, nous proposons une artérialisation de la veine dorsale profonde. Les frondes ou garrots synthétiques, les plicatures des corps caverneux, peuvent être utilisées comme techniques complémentaires. Elles n'ont pas fait toutefois la preuve de leur efficacité à long terme. Toutes ces techniques doivent obligatoirement être menées sous microscope d'opération avec une micro-instrumentation adaptée.

\section{Résultats}

Ceux-ci sont très difficiles à établir, car les séries sont disparates, non actualisées, le suivi trop court, les techniques différentes, les indications non standardisées. L'appréciation est le plus souvent subjective, basée sur un simple interrogatoire.

\section{Pathologie artérielle pure}

Quoi qu'il en soit, les résultats pour la pathologie artérielle pure se situent entre 65 et $80 \%$ de succès. En ce qui concerne les résultats personnels, le succès est établi avec une érection qui dure plus de 5 minutes, suffisamment rigide pour autoriser une pénétration vaginale normale.

Les échecs apparaissant au cours des deux premières années, sont le fait de mauvaises indications ou de fautes techniques, en particulier chez des hommes âgés (tableau 1).

Tableau 1 : Résultats de la chirurgie reconstructrice en cas de pathologie artérielle pure

\begin{tabular}{lccc}
\hline & Nombre & Recul & Succès \\
\hline AUSTONI (1) & 87 & $?$ & $77 \%$ \\
CRESPO (3) & 390 & 1 à 13 ans & $73 \%$ \\
VIRAG (22) & 151 & 36 mois & $74 \%$ \\
GOLDSTEIN (6) & 150 & 6 mois à 4 ans & $80 \%$ \\
SARRAMON (19) & 37 & 4 mois à 8 ans & $88 \%$ \\
\hline
\end{tabular}

\section{Lésions artérielles et veineuses associées} dites mixtes

On trouve peu de publications sur ce sujet car le recul est encore trop court; il semble pourtant que cette double pathologie soit très fréquente (tableau 2).

Tableau 2 : Résultats de la chirurgie reconstructrice en cas de lésions mixtes

\begin{tabular}{lccc}
\hline & Nombre & Recul & Succès \\
\hline CRESPO (3) & 38 & 1 et 3 ans & $71,3 \%$ \\
SARRAMON (18) & 27 & 2 à 42 mois & $82 \%$ \\
\hline
\end{tabular}

\section{Pathologie veineuse}

De nombreuses publications rapportent les résultats de la chirurgie des fuites veineuses. Ils sont très variables et ceci en fonction du recul et aussi des techniques. Malgré les trop courtes séries actuelles, il semble que le meilleur procédé soit l'artérialisation de la veine dorsale profonde (22). (tableau 3).

Tableau 3 : Résultats de la chirurgie reconstructrice en cas de pathologie veineuse

\begin{tabular}{lcccc}
\hline & Nombre & Technique & Recul & Succès \% \\
\hline AUSTONI (1) & 27 & $\begin{array}{c}\text { ligature VDP } \\
\text { spongiolyse } \\
\text { plicature C.C. }\end{array}$ & immédiat & 83 \\
suspension C.C. & & \\
KNOLL (10) & 12 & $\begin{array}{c}\text { Artérialisation } \\
\text { V.D.P. }\end{array}$ & $?$ & 42 \\
SARRAMON (18) & 13 & artérialisation & 2 à 42 mois & 91 \\
ROSSI (17) & 26 & gartot \\
& prothétique & $12-25$ mois & 61,5 \\
& & & \\
\hline
\end{tabular}

\section{PROTHESES}

Il existe sur le marché une multitude de prothèses.

On distingue essentiellement deux catégories : les prothèses semi-rigides et les prothèses gonflables. Le choix est fonction des patients, de leurs désirs. L'indication doit tenir compte de l'âge, de la volonté du couple qui doit être instruit des possibilités et des performances des différents modèles sans négliger les incidents techniques ou les risques infectieux. Nous nous bornerons à décrire seulement les plus couramment utilisées.

\section{Prothèses semi-rigides}

Le matériau qui les compose est un élastomère de silicone, qui a fait la preuve de sa bonne tolérance tissulaire, et qui donne une rigidité suffisante avec une flexibilité adéquate.

Prothèse de Small-Carrion (20) : les plus populaires aux USA, elles possèdent une bonne rigidité, leur portion proximale est fusiforme, épouse très bien la racine des corps caverneux. Il existe 16 tailles différentes, 3 diamètres : $9,11,13 \mathrm{~cm}$ et 9 longueurs de 12 à $21 \mathrm{~cm}$. Elles s'adaptent avec précision à toutes les tailles de verge, linnconvénient est qu'il faut posséder une batterie complète.

Prothèse de Subrini (21) : élastomère de silicone, partie proximale souple qui peut être taillée à la dimension de la verge, deux diamètres disponibles $10 \mathrm{et} 11 \mathrm{~cm}$; de plus, on peut en réduire la longueur par recoupe adaptée dans la partie proximale. Très bien tolérée, avec possibilité d'érections sur la prothèse si le tissu caverneux n'a pas été détruit, le coût est très peu élevé.

Prothèse de Finney "Flexi rods" (4) : leur partie proximale est effilée, on peut la recouper selon la longueur des corps caverneux ; la partie moyenne est flexible, elle correspond à la région sous pubienne, c'est elle qui donne la mobilité. La partie distale qui intube les corps caverneux dans leur portion externe est rigide. Les dimensions 
varient en diamètre et longueur : 9 à $14 \mathrm{~mm}$ et 7 à $13 \mathrm{~cm}$. En règle générale on utilise $12 \mathrm{~mm}$ de diamètre et $12 \mathrm{~cm}$ de longueur.

Prothèse Eska Jonas (8) : en silicone, elles ont la même consistance, elle peuvent être recoupées dans leur portion distale, elles possèdent en leur centre une armature en fil d'argent torsadé qui permet d'orienter le pénis. Proposées avec deux diamètres : 9,5 et $11 \mathrm{~mm}$ et 6 longueurs de 17 à $22 \mathrm{~cm}$.

Prothèse AMS 600 malléable : elles peuvent être diminuées dans leur diamètre en retirant une enveloppe de silicone, et allongées par des embouts de 1, 2 et $3 \mathrm{~cm}$ de longueur. Elles sont orientables grâce à une âme en acier (fig. 5).

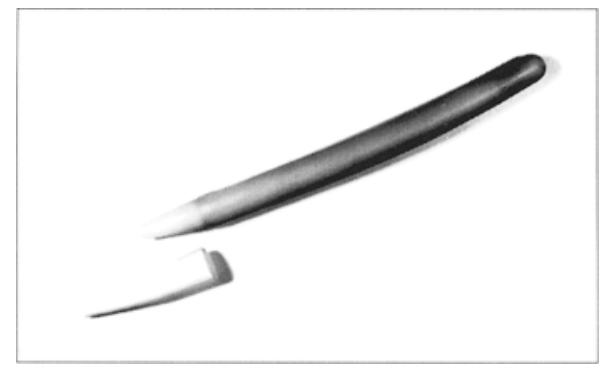

Fig. 5 : Prothèse semi-rigide armée

\section{Prothèses gonflables}

Prothèses duraphase et omniphase :De toutes les prothèses ce sont les plus physiologiques, car elles donnent à la verge un aspect sub normal à l'état de flaccidité. Il en existe deux types différents : celles dont le réservoir est séparé et celles qui l'ont incorporé et pré-rempli.

Prothèse de Scott AMS 700 Ultrex : dernière née, très simplifiée dans son fonctionnement, possède un système de valves très fiables ainsi que des embouts qui modifient leur longueur à la demande, ce sont elles qui donnent à la verge la meilleure turgescence et rigidité, elles permettent aussi un allongement de la verge ; elles sont délicates à placer du fait de la complexité des circuits. Le principal écueil est leur prix beaucoup trop élevé (fig. 6).

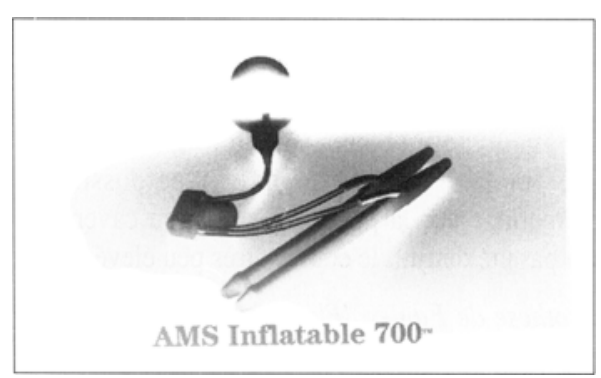

Fig. 6 : Prothèse gonflable ULTREX

Dynaflex $(T m)$ : prothèse avec deux cylindres autogonflables, elle a remplacé l'Hydroflex. Aussi facile à placer que les prothèses semi- rigides, elle donne une tumescence satisfaisante et une rigidité convenable, le diamètre est de 11 , 13 et $15 \mathrm{~mm}$, la pompe est facile à actionner en amont du gland, le dégonflage se fait par simple flexion du pénis.

Mentor : c'est une prothèse hydraulique qui comporte un système unique : réservoir et pompe implantable directement au niveau de scrotum sans intervention rigide abdominale ; cette prothèse est à base de polyuréthane qui est très bien toléré et qui résiste à la distension sans risque d'anévrysme. L'accès à la pompe et à la valve est simple et permet un fonctionnement aisé du système.

\section{INDICATIONS}

Elles doivent être particulièrement mesurées, car une fois la prothèse implantée, on ne possède aucun autre recours pour traiter l'impuissance. Il faut prendre l'avis du couple et au besoin d'un psychiatre avant l'implantation. Les meilleures indications sont représentées par les échecs des autres procédés en particulier chez les vasculaires. On les proposera après traumatisme neurologique périphérique, accident de la route, la chirurgie carcinologique du rectum ou de la vessie et prostate, chez les vasculaires et diabétiques, enfin, certains paraplégiques, certaines fibroses extensives des corps caverneux (maladie de La Peyronie, suites du priapisme).

\section{RESULTATS}

Les résultats globaux de tous types de prothèses confondus se situent entre 85 et $90 \%$ de patients satisfaits (tableaux 4 et 5 ). Les résultats immédiats sont favorables. Par contre, si on analyse le résultat cosmétique et psychologique par un questionnaire détaillé, il est frappant de noter que $78 \%$ se voient pleinement satisfaits. L'insatisfaction se retrouve au niveau de la partenaire qui regrette parfois un système prothétique.

Tableau 4 - Résultats des prothèses semi-rigides

\begin{tabular}{lccc}
\hline & Nombre & Succès & $\begin{array}{c}\text { Complications } \\
\text { majeures }\end{array}$ \\
\hline SMALLL 1979 (20) & 160 & $95 \%$ & $2.5 \%$ \\
SUBRINI 1982(21) & 283 & $95 \%$ & $6 \%$ \\
FINNEY (4) & 100 & $95 \%$ & $5 \%$ \\
JONAS (8) & 29 & $89 \%$ & $10 \%$ \\
\hline
\end{tabular}

Tableau 5 - Résultats des prothèses gonflables

\begin{tabular}{lccc}
\hline & Nombre & Succès & $\begin{array}{c}\text { Complications } \\
\text { majeures }\end{array}$ \\
\hline FURLOW (5) & 210 & $98,5 \%$ & $3 \%$ \\
MERILL (15) & 206 & $98 \%$ & $12 \%$ \\
Mc LAREN (14) & 500 & $88 \%$ & $20,5 \%$ a \\
a : + problèmes mécaniques chez 50\% & \\
\hline
\end{tabular}

Qu'il s'agisse de prothèses semi-rigides ou gonflables, les résultats sont superposables. Les échecs sont surtout d'ordre psychologique, ou le fait de complications. On retiendra toutefois un taux de complications supérieur pour les prothèses gonflables.

Les complications sont relativement rares avec les prothèses semi-rigides; des anomalies de fonctionnement peuvent se rencontrer dans le cas de prothèses gonflables.

Les complications précoces sont l'érosion avec rejet septique (Gram-), en particulier chez le diabétique. La gêne et la difficulté d'utilisation peuvent parfois amener à un changement, par exemple prothèse semi-rigide pour prothèse gonflable. Les complications tardives : infection et érosion secondaire $($ Gram + ) se voient dans $14 \%$ des cas, avec transfixion du gland, plus rarement érosion de l'urèthre. Les pannes mécaniques avec les nouvelles prothèses sophistiquées sont évaluées à $20 \%$ (fuite de liquide, altération de la pompe, en particulier fibrose, réservoir ou cylindre disparates). Elles nécessitent l'ablation de la totalité du matériel avec risque de voir des suppurations chroniques. Les traitements de tous ces problèmes sont très spécialisés et demandent une excellente connaissance des différentes prothèses et de leur fonctionnement.

Les changements de prothèse ne posent aucun problème (14) s'il n'y a pas d'intervalle libre, par contre, s'il y a eu sepsis, on conseille actuellement un traitement local antibiotique énergique et un remplacement rapide de la prothèse pour éviter la fibrose. Les résultats de ces réimplantations dépendent en effet de l'état de fibrose des corps caverneux. On a pu à ce sujet proposer l'excision du tissu fibreux à l'uréthrotome ou au ciseau de Mayo et le remplacement d'une partie de l'albuginée par des plaques de Gortex ou de Dacron. Les échecs sont de l'ordre de $50 \%$ dans ces cas-là.

\section{DISCUSSION}

La chirurgie de revascularisation artérielle des corps caverneux a acquis cette dernière décennie ses lettres de noblesse (18). Les indications sont actuellement bien établies. Chaque fois qu'une artère dorsale le permet, le pontage épigastricodorsal donne d'excellents résultats à long terme. On connaît anatomiquement la possibilité de revasculariser le corps caverneux à partir des branches collatérales profondes de l'artère dorsale (9). La revascularisation directe des artères caverneuses est d'indication plus rare car dans la grande majorité des cas, ces artères sont obstruées et de très petit calibre ; on peut toutefois réserver ce procédé aux séquelles de traumatisme. 
L'artérialisation de la veine dorsale profonde est une bonne intervention à condition de disséquer sous microscope la veine dorsale profonde et de ponter en regard de 2 à 3 veines émissaires (14) que l'on verra plonger dans le corps caverneux. La ligature d'amont est nécessaire, mais ne prévient pas a priori le risque d'hypervascularisation du gland. Il faut se méfier d'artères épigastriques de gros calibre ( $2 \mathrm{~mm}$ de diamètre). Ce dernier procédé peut être utile pour traiter les patients artériels purs pour lesquels on n'a aucune possibilité de pontage artériel. Mais à notre avis, il parait le meilleur procédé pour traiter les fuites veineuses.

Toutes ces techniques sont longues, minutieuses, nécessitent un entrainement régulier à la microchirurgie ; les anastomoses de si petits vaisseaux ne souffrent pas l'à-peu-près. La thrombose aiguë est la sanction immédiate et irréversible d'une faute technique. Enfin, il faut connaitre la fréquence des lésions mixtes qui nécessitent un traitement mixte, qui aura pour but d'accroître le débit artériel et de freiner le retour veineux. Tous ces procédés justifient à notre avis un traitement anticoagulant au long cours, de manière à obtenir une isocoagulation stable.

Les échecs sont finalement rares. Ils sont le fait de difficultés techniques, artère épigastrique trop fine, se divisant trop tôt ou athéromateuse. Actuellement, nous ne revascularisons plus après 65 ans. Cette chirurgie est très encourageante, elle ne ferme pas les portes à d'autres méthodes comme les auto-injections de drogues vasoactives ou la mise en place de prothèses. A ce sujet, certains de nos patients ont pu obtenir un résultat excellent par l'association des deux procédés.

En ce qui concerne les prothèses péniennes, les résultats sont excellents dans la mesure où l'indication est mesurée, le patient bien averti et le type de prothèse bien choisi. Une prothèse donne une érection cosmétiquement excellente mais non physiologique. Le refus de la partenaire ou de certains patients correspond à un manque d'information dans la période pré-opératoire.

Au total, la chirurgie de limpuissance est devenue une véritable spécialité, elle demande une connaissance globale de la pathologie générale, une appréciation rigoureuse dans la décision thérapeutique, une maîtrise de la microchirurgie et une pratique régulière d'implantation des différentes prothèses.

\section{REFERENCES}

1 - Austoni E., Cazzaniga A., Colombo F., Toia G., Pisani F. Long term follow-up after double epigastrico-dorsal end to end anastomosis, orthoflow and antiflow. Int. J. Impotence Res., 1990, suppt $2: 440-441$.

2- Bove D.A., Soltanik E.M., Farell G.R., Crespo E.L. Combinated penile vascular impotence. Int. J. Impotence Res. 1990, suppt 2: 394.

3 - Crespo E.L., Soltanik E.M., Bove D.A., Farell G.R. 13 years of experience in microvascular surgery for pure arterial impotence. Int. J. Impotence Res., 1990, suppt $2: 395$.

4 - Finney R.P. Finney flexirod prosthesis. Urology, 1984, $23: 79$.

5 - Furlow W.L., Knoll L.D., Motlex R.C. Clinical experience implanting an implantable penile prosthesis with a controlled expansion cylindra. Proceedings of the third biennal world meeting on impotence, Boston, $1988: 216$.

6 - Goldstein I. Contemporary management of impotence and infertility. William Wilkin eds Baltimore, 1988 : p. 171.

7 - Hauri D.A. A new operative technique in vasculogenic erectile impotence. World J. Urol., 1986, $4: 237$.

8 - Jonas U. Five years experience with the silicone silver penile prosthesis improvements and new developments. World J. Urol., 1983, $1: 251$.

9 - Juskiewenski S., Vaysse Ph., Moscovici J., Mamoundi S., Bouissou P. A study of the arterial blood supply to the penis. Anat. Chir., 1982, 4 : 101-107.

10 - Knoll L.D., Furlow W.L., Benson R.C. Deep dorsal vein arterialization in the management of cavernosal venous leakage. Proceedings of the 86 th annual meeting of the American Urological Association, J. Urol., 1991, 145 : 526.

11 - Konnak J.W., Ohl D.A. Microsurgical penile revascularization using the central corporeal penile artery. J. Urol., 1989, $142: 305$.

12 - Lewis R.W. Venous ligation surgery for venous leakage. Int. J. Impot. Res., 1990, 2 : 1-19.

13 - Lowsley O. Impotence in man. South Med J. , 1953, $12: 1091$.

14 - Mc Laren R.H., Lewis R.W. The reoperated penile implant. Int. J. Impotence Res., 1990, suppt $2: 463$ - 464 .

15 - Merill D.C. Clinical experience with Mentor inflatable prosthesis in 206 patients. Urology, 1986, $27: 1985$.
16 - Michal V., Kramer R., Hejhal L. Direct arterial anastomosis on corporeal cavernosa penis in the therapy of erectile impotence. Rozal Chir., 1973, $52: 287$.

17 - Rossi D., Coulange C., Serment G., Hermanowicz M., Chiapello A., Ducassou J. Resultats du traitement chirurgical de l'impuissance par fuite veineuse par mise en place du garrot veineux prothétique. Ann. Urol., 1990, 24 : 306.

18 - SARRAMON J.P., BOCCALON H., ROSSIGNOL G. Penile revascularization in Urology, L.V. Wagenchnecht ed, Thieme, 1985.

19 - Sarramon J.P., Rischmann P., Lemba P., Boumel P., Pujol P. Microsurgery reconstruction for pure vascular impotence. J. Urol. 1990, 143: 303 A.

20 - Small M.P., Carrion H., Gordon J.A. Small Carrion prosthesis. New implant for management of impotence. Urol., 1975, 5: 479.

21 - Subrini L. Subrini penile implants : surgical, sexual and physiological results. Eur. Urol., 1982, $8: 22$.

22 -Virag R. Revascularization penis. In : Management of male impotence, Bennet A.M. ed., Williams Wilkins, Baltimore, 1982.

23 - Wespes E., Corbusier A., Delcour C., Vandenbosch G., Struyven J., Schulman C.C. Deep dorsal vein arterialization in vascular impotence. Brit. J. Urol., 1989, 64 : 534-538.

24 - Wooten J.S. Ligation of the dorsal vein of the penis as a cure for atonic impotence. Texas Med J., 1902, $18: 325-328$.

RESUME : Mise au point actualisée des différentes possibilités chirurgicales pour traiter l'impuissance en 1991. Les techniques de revascularisation artérielle microchirurgicales donnent entre 70 et $87 \%$ d'excellents résultats avec des reculs de plus de 3 ans. Pour la pathologie veineuse, il semble que l'artérialisation de la veine dorsale profonde donne les meilleurs résultats et les plus durables. Les prothèses péniennes bénéficient des progrès constants de la technologie, les prothèses gonflables sont les mieux tolérées, les défauts mécaniques sont actuellement rares, mais leur prix demeure élevé. La chirurgie vasculaire doit toujours être tentée en priorité, car elle est physiologique, et ne compromet pas les autres procédés : auto-injection, vacuum ou prothèse pénienne. Mots clés : Impuissance vasculaire, Revascularisation pénienne, Prothèses. Andrologie, 1991, 1 : 145-148. 